\title{
Efficient quantum modeling of inelastic interactions in nano-devices
}

\author{
Y. Lee,${ }^{1, *}$ M. Lannoo, ${ }^{1}$ N. Cavassilas, ${ }^{1}$ M. Luisier, ${ }^{2}$ and M. Bescond ${ }^{1, \dagger}$ \\ ${ }^{1}$ IM2NP, UMR CNRS 7334, Aix-Marseille Université, Technopôle de Château-Gombert, \\ Bâtiment Néel, 60 Rue Frédéric Joliot Curie, 13453 Marseille, France \\ ${ }^{2}$ Integrated Systems Laboratory, ETH Zürich, 8092 Zürich, Switzerland
}

\begin{abstract}
This work presents an efficient direct quantum method to model inelastic scattering in nanoelectronic structures including degenerate band extrema. It couples the Born series expansion of the non-equilibrium Green's function (NEGF) to an analytic continuation based on the Padé approximant technique. Using a two-band $\mathrm{k} \cdot \mathrm{p}$ Hamiltonian, we analyze the electron transport through a linear chain in presence of both optical and acoustic phonons. Results are consistently compared with the usual, computationally expensive, self-consistent Born approximation (SCBA). We find that our approach provides a much better convergence for both types of phonons in presence of strong multi-band coupling. The calculation of the current to the $5^{\text {th }}$ order in the interactions is sufficient to reproduce the influence of all considered phonon interactions. We also show that the method can be applied to the calculation of the density of carriers which depicts however a slower convergence rate than the current. The capability to efficiently calculate both current and charge density represents a clear advantage in a context of increasing request for atomistic quantum simulations.
\end{abstract}

\section{INTRODUCTION}

The great progress of semiconductor industry over the last 30 years has been accompanied by an impressive development of the quantum transport modeling of nanostructures and devices ${ }^{1}$. Indeed experimental advances in mesoscopic physics and innovations in nanoelectronics require the use of sophisticated quantum transport simulation tools capable to capture the physical properties of such systems. One of the most difficult part in quantum transport modeling is to manage the computational burden induced by the treatment of inelastic interactions between electrons and other particles like phonons or photons. Unfortunately, these interactions are found to have a non-negligible influence and even in some cases can be at the origin of the working principle of the device. This is for instance the case of electron-photon interaction in solar cells.

Several quantum approaches have been intensively developed during the last decade to address this issue $^{2-6}$. Among them the non-equilibrium Green's function (NEGF) formalism appears as one of the most efficient methods to include interactions in quantum transport codes using the concept of self-energies ${ }^{7}$. This approach has been successfully applied to various nano-structures and devices, including quantum $\operatorname{dots}^{8,9}$, molecular junctions ${ }^{10}, 2 \mathrm{D}$ materials ${ }^{11}$, resonant tunneling diodes ${ }^{12}$, semiconductor transistors ${ }^{13-19}$ and solar cells ${ }^{20-22}$. Despite significant improvements the NEGF method still requires heavy computational resources when dealing with interactions in realistic nanostructures. Indeed electron-phonon/photon interactions are usually treated within the self-consistent Born approximation (SCBA) through an iterative resolution of the Dyson equation. Self-consistency is often believed to be "the price to pay" to obey all conservation laws and to obtain physically meaningful solutions. However, we recently demonstrated that this is not the case. The calculated self-energies must not be necessarily obtained through a self-consistent scheme, but more importantly via the derivative of a functional $\phi$ in the picture of Luttinger and Ward ${ }^{23,24}$. Considering a lowest order approximation (LOA) for the interaction we first demonstrated in Ref. 25 that self-consistency is sufficient, but not necessary to conserve the current. Furthermore, in Ref.26 we have proposed an infinite family of non-self-consistent, but conserving approaches based on an extension of the LOA relying on the Green's function Born series. This extended LOA technique coupled with an analytic continuation of Padé approximants ${ }^{27,28}$ has been shown to provide excellent expectation values of the current (at least very close to those obtained with SCBA). The first order version of the analytically continuated LOA has been then applied to the calculation of current characteristics with phonon scattering in double-gate ${ }^{29}$ and nanowire ${ }^{30}$ n-type transistors.

The aim of the present work is twofold. Firstly, we want to precisely describe the computational scheme of the so-called analytically continuated LOA technique and to assess its limits in providing accurate current expectation values in presence of phonon scattering. To do so we will consider an ideal 1D system described by different Hamiltonians. Two-band $\mathrm{k} \cdot \mathrm{p}$ models will be used to assess the validity of the approach when multi-band transport becomes predominant. The calculation of the charge density is also an important point in the context of nanostructure quantum transport modeling. Indeed, the calculation of the current requires the determination of the electrostatic potential which is itself computed, in most cases, via a self-consistent loop between the charge density and the Poisson equation. We will therefore describe the computation of charge densities within the analytically continuated LOA approach and will apply it to the Hamiltonians previously mentioned. Such a validated approach represents a significant reduction of the computational complexity with respect to the SCBA since 
it provides a general recipe to efficiently calculate both current and charge densities in non-equilibrium quantum systems.

The rest of the paper is organized as follows: In Sec.II we will describe the implementation of the LOA starting from the SCBA. Higher order LOA and analytic continuation techniques will be presented in the sub-sections for both current and charge density in the presence of electron-phonon interactions (acoustic and optical). Section III will show the details of the computational implementation of this scheme. Section IV will present the various results for each types of Hamiltonian and phonon interaction intensity. Finally, Section V will summarize the key findings.

\section{THEORETICAL FRAMEWORK}

In this section, theoretical details of the LOA are introduced starting from the comparison with the usual SCBA. In particular we focus on the physical and computational advantages of the LOA over the SCBA. Thereafter, based on this scheme we will provide the generalized LOA method to calculate the interacting Green's function to any order. In addition, we will apply analytic continuations based on the Padé approximants for the calculation of currents and charge densities.

\section{A. Self-Consistent Born Approximation}

Inelastic interactions in the NEGF framework are usually treated using the SCBA in which scattering processes are treated via self-energy contributions ${ }^{13-15,19}$. The calculation of these self-energies is performed using the Dyson equation, which can be expressed in the simplified matrix notation $\left[1=\left(r_{1}, t_{1}\right), g_{0} \Sigma[G] G=\right.$ $\left.\int d 2 \int d 2^{\prime} g_{0}(1 ; 2) \Sigma\left(2 ; 2^{\prime}\right) G\left(2^{\prime} ; 1^{\prime}\right)\right]$ as

$$
G=g_{0}+g_{0} \Sigma[G] G
$$

where $g_{0}$ is the non-interacting (or ballistic) Green's function, $\Sigma$ is the self-energy and $G$ is the fully interacting Green's function ${ }^{25}$. The resolution of this equation usually requires an iterative scheme which can be written as

$$
G_{N}=\left[g_{0}^{-1}-\Sigma\left[G_{N-1}\right]\right]^{-1}
$$

where $G_{N}$ is the Green's function at the $N^{t h}$ iteration. It depends on the self-energy calculated from the Green's function at the previous iteration with $G_{0}=g_{0}$. When the iterative process converges, i.e. $G_{N}=G_{N-1}$, it leads to the SCBA, which has been shown to be current conserving along the device ${ }^{31}$. However, truncated series expansions of Eqs. $(1-2)$ do not necessarily preserve these conservation laws. Therefore, the SCBA should be treated with specified convergence criteria to obtain the conserving results. This iterative character is definitively the bottleneck in real applications since it may become computationally very expensive. For example, the atomistic full-band simulator described in Ref.15 requires heavy computational resources ( $>3000 \mathrm{CPUs}$ ) with up to 100 SCBA iterations to reach good convergences.

\section{B. Lowest Order Approximation (LOA)}

Choosing a proper self-energy is the most important task in the NEGF framework since this quantity should accurately describe the physical properties of the system and fulfill the conservation laws. It has been shown that a $\Phi$-derivable self-energy in the Luttinger-Ward picture $^{23,24}$ guarantees the conservation laws. The SCBA self-energy is $\Phi$-derivable, but it requires a lot of iterations to converge, as mentioned previously. Therefore, it is of interest to devise an alternative approach satisfying the conservation laws, while avoiding the iterative scheme. The simplest LOA is a one-shot approach that meets these requirements. To build up the LOA one can also start from the Dyson equation and write

$$
G=g_{0}+g_{0} \Sigma[G] G=g_{0}+\delta G .
$$

We can consider the non-interacting (or ballistic) Green's function $g_{0}$ as an unperturbed term and the other one, $g_{0} \Sigma[G] G$, as a perturbation $\delta G$. By injecting these terms $g_{0}+\delta G$ into the fully interacting Green's function $G$ of Dyson equation and applying a first-order Taylor series expansion to the interacting self energy $\Sigma\left[g_{0}+\delta G\right]$, we get $^{25}$

$$
\begin{aligned}
G & =g_{0}+g_{0} \Sigma[G] G \\
& =g_{0}+g_{0} \Sigma\left[g_{0}+\delta G\right]\left(g_{0}+\delta G\right) \\
& \approx g_{0}+g_{0} \Sigma\left[g_{0}\right] g_{0}+g_{0} \Sigma\left[g_{0}\right] \delta G \\
& +g_{0} \frac{\partial \Sigma[G]}{\partial G} \delta G g_{0}+g_{0} \frac{\partial \Sigma[G]}{\partial G} \delta G^{2}
\end{aligned}
$$

where the interacting self energy $\Sigma\left[g_{0}+\delta G\right]=\Sigma\left[g_{0}\right]+$ $\frac{\partial \Sigma[G]}{\partial G} \delta G$. By restricting Eq. (4) to the first-order in interaction, we obtain the LOA Green's function $g_{L O A}$ that only depends on the non-interacting Green's function $g_{0}$

$$
g_{L O A}=g_{0}+g_{0} \Sigma\left[g_{0}\right] g_{0}
$$

This Green's function does not violate the conservation laws since the self-energy $\Sigma\left[g_{0}\right]$ is found to be $\Phi$-derivable. Bescond et al. in Ref. 30 have shown that $g_{L O A}$ is equivalent to the $G_{1}$ of the SCBA scheme in Eq. (2) when the perturbation term is very small and that it only includes one interaction process. Finally, this scheme can be the basic building block to generalize LOA for calculating the interacting Green's functions $g_{N^{t h} L O A}(N>1), N$ defining the order in the interaction, which is given in the next section. 


\section{Generalization of the Lowest Order Approximation}

Mera et al. in Ref. 26 have shown that one can construct the Born series of the Green's function starting with the basic building block $\Delta g_{1}=g_{0} \Sigma\left[g_{0}\right] g_{0}$ of the LOA. The Green's function for the Born series up to order $N$ in the interaction $\left(g_{N} \equiv g_{N^{t h} L O A}, N>1\right)$ can be generalized as

$$
g_{N}=g_{0}+\sum_{n=1}^{N} \Delta g_{n}
$$

where $\Delta g_{n}$ is the term of order $n$ in the strength of the interaction. In this paper we focus on the case of the electron-phonon interaction where the self-energy of the interaction is linear in the Green's function and also in the strength of the interaction. This means that $\Sigma[G]$ can also be expanded in a Born series for which the term of order $n$ is given by

$$
\Sigma_{n}=\Sigma\left[\Delta g_{n-1}\right] .
$$

Since only the processes of $N$ interactions should be included for the Green's function $g_{N}$ to satisfy the conservation laws, we can then discard higher order perturbation terms. We can now generalize the Born series starting from the Dyson equation (1) and expressing the fact that the term of order $N$, i.e. $\Delta g_{N}$, results from all products of that order contained in $\Sigma[G] G$. We thus get, for $N \geqslant 1$

$$
\Delta g_{N}=g_{0} \sum_{n=0}^{N-1} \Sigma_{N-n} \Delta g_{n}
$$

with

$$
\Delta g_{1}=g_{0} \Sigma\left[g_{0}\right] g_{0},
$$

and

$$
g_{N}=g_{N-1}+\Delta g_{N} .
$$

By using these Eqs. $(8-10)$, we obtain the carrier density series $\boldsymbol{\rho}_{N}^{(j)}=\boldsymbol{\rho}^{(j)}\left(g_{N}\right)$ and current series $\mathcal{I}_{N}=$ $\mathcal{I}\left(g_{N}\right)$ to any order in the interaction

$$
\boldsymbol{\rho}_{N}^{(j)}=\boldsymbol{\rho}_{0}^{(j)}+\sum_{n=1}^{N} \Delta \boldsymbol{\rho}_{n}^{(j)}, \mathcal{I}_{N}=\mathcal{I}_{0}+\sum_{n=1}^{N} \Delta \mathcal{I}_{n}
$$

where $\Delta \boldsymbol{\rho}_{n}^{(j)}=\boldsymbol{\rho}_{n}^{(j)}-\boldsymbol{\rho}_{n-1}^{(j)}$ and $\Delta \mathcal{I}_{n}=\mathcal{I}_{n}-\mathcal{I}_{n-1}$ are defined as the difference between the expectation values of the $n^{\text {th }}$ and $(n-1)^{t h}$ order LOA, and $\boldsymbol{\rho}_{0}^{(j)}$ and $\mathcal{I}_{0}$ are the ballistic expectation values. It has to be noted that $\boldsymbol{\rho}_{N}^{(j)}$ represents a carrier density of a local site $(j)$ in the real space. It is defined on each point of the system and then computationally detailed by a vector whose dimension depends on the mesh discretization and the size of the studied system.

This series of current and charge density guarantees the conservation laws by construction. However, it may converge or diverge according to the strength of the considered interaction. Therefore, in the next section we also present Padé approximants to analytically continue this series in the case of divergence.

\section{Padé Approximants}

Some of us has demonstrated ${ }^{26}$ that any current expectation values can be calculated by using the Born series, while preserving the conservation laws. However, since this series has a radius of convergence depending on the intensity of the interaction, the energy of the particle, etc., Padé approximants have been introduced as an efficient remedy to treat divergent behavior ${ }^{27,28,32}$. Padé approximants represent a relevant method in the way they can provide several sets of rational approximations.

Considering a function $F(z)$, the Taylor series is represented as $F_{N}(z)=\sum_{k=0}^{N} \frac{F^{(k)}}{k !} z^{k}$ where $F^{(k)}$ is the $k^{t h}$ derivative of $F$. The Padé approximant to this function $F(z)$ is defined as a rational function $F_{l / m}(z)$ in a fractional form composed of two polynomials $L_{l}(z), M_{m}(z)$ such $\mathrm{as}^{33}$

$$
\begin{aligned}
F_{l / m}(z) & =\frac{L_{l}(z)}{M_{m}(z)} \\
& =\frac{l_{0}+l_{1} z+l_{2} z^{2}+\cdots+l_{l} z^{l}}{1+m_{1} z+m_{2} z^{2}+\cdots+m_{m} z^{m}} .
\end{aligned}
$$

By using the relation $F_{N}(z) M_{m}(z)-L_{l}(z)=0$, we can determine the unknown coefficients $l_{0}, \ldots, l_{l}$ and $m_{1}, \ldots, m_{m}$ up to $l+m$ order. Here, the Padé table of the arbitrary function $F(z)$ constructed up to the second order is given as

$$
F_{l / m}=\left(\begin{array}{ccc}
f_{0} & f_{0}+f_{1} z & f_{0}+f_{1} z+f_{2} z^{2} \\
\frac{f_{0}}{1-f_{1} z / f_{0}} & \frac{f_{0}+\left(f_{1}-f_{0} f_{2} / f_{1}\right) z}{1-f_{2} z / f_{1}}
\end{array}\right)=\left(\begin{array}{lll}
f_{0 / 0} & f_{1 / 0} & f_{2 / 0} \\
f_{0 / 1} & f_{1 / 1} \\
f_{0 / 2} &
\end{array}\right),
$$

where it is noticeable that the first row of the table, i.e.

$F_{l / 0}(l \leqslant 2)$, is exactly equivalent to the Taylor series of 
the function $F(z)$. Therefore, when the function can be represented by a Taylor series, we can use several rational forms of the Padé approximant. For example, once we apply Eq. (11) for the charge density and current series to the first row of the Padé table (13) for the arbitrary function such as $f_{0}=\boldsymbol{\rho}_{0}^{(j)}, f_{1} z=\Delta \boldsymbol{\rho}_{1}^{(j)}$ and $f_{2} z^{2}=$ $\Delta \boldsymbol{\rho}_{2}^{(j)}$, we can reconstruct the Padé table for the charge and current as

$$
\begin{aligned}
& \boldsymbol{\rho}_{l / m}^{(j)}=\left(\begin{array}{c}
\boldsymbol{\rho}_{0}^{(j)} \\
\frac{\boldsymbol{\rho}_{0}^{(j)}}{1-\Delta \boldsymbol{\rho}_{1}^{(j)} / \boldsymbol{\rho}_{0}^{(j)}} \\
\frac{\boldsymbol{\rho}_{0}^{(j)}}{1-\Delta \boldsymbol{\rho}_{1}^{(j)} / \boldsymbol{\rho}_{0}^{(j)}+\left(\Delta \boldsymbol{\rho}_{1}^{(j)} / \boldsymbol{\rho}_{0}^{(j)}\right)^{2}-\boldsymbol{\rho}_{2}^{(j)} / \boldsymbol{\rho}_{0}^{(j)}}
\end{array}\right. \\
& \mathcal{I}_{l / m}=\left(\begin{array}{c}
\mathcal{I}_{0} \\
\frac{\mathcal{I}_{0}}{1-\Delta \mathcal{I}_{1} / \mathcal{I}_{0}} \\
\frac{\mathcal{I}_{0}}{1-\Delta \mathcal{I}_{1} / \mathcal{I}_{0}+\left(\Delta \mathcal{I}_{1} / \mathcal{I}_{0}\right)^{2}-\mathcal{I}_{2} / \mathcal{I}_{0}}
\end{array}\right.
\end{aligned}
$$

\section{COMPUTATIONAL IMPLEMENTATION}

This section describes in detail the calculation of carrier densities and currents by using the first and higher order LOA. In particular, we apply the formalism in the case of electron-phonon coupling.

\section{A. Calculation of Green's Functions by Implementing the LOA Algorithm}

Let us begin with the definition of the retarded noninteracting/ballistic Green's function $g_{0}^{r}$ connected to two contact reservoirs (labelled $\mathrm{L}$ for the left contact and $\mathrm{R}$ for the right contact) such as

$$
g_{0}^{r}=\left[E I-H-\Sigma_{L}^{r}-\Sigma_{R}^{r}\right]^{-1},
$$

where $I$ is the identity matrix, $\Sigma_{L / R}^{r}$ are the retarded self-energy for the left/right device contacts ${ }^{34}$ and $H$ represents the Hamiltonian of the active region. The corresponding lesser/greater Green's functions are defined as

$$
g_{0}^{\lessgtr}=g_{0}^{r}\left(\Sigma_{L}^{\lessgtr}+\Sigma_{R}^{\lessgtr}\right) g_{0}^{a},
$$

where $g_{0}^{a}$ is the advanced Green's function obtained from the Hermitian conjugate of its retarded counterpart $\left(g^{a}=g^{r \dagger}\right)$ and each $\Sigma \lessgtr$ function is the lesser/greater selfenergy

$$
\begin{gathered}
\Sigma_{L / R}^{<}=+i \Gamma_{L / R} f_{L / R}, \\
\Sigma_{L / R}^{>}=-i \Gamma_{L / R}\left(1-f_{L / R}\right),
\end{gathered}
$$

$f_{L / R}$ being the Fermi-Dirac distribution function of the left and right reservoirs, respectively and $\Gamma_{L / R}$ the broadening function calculated according to the following
$\left.\begin{array}{cc}\boldsymbol{\rho}_{0}^{(j)}+\Delta \boldsymbol{\rho}_{1}^{(j)} & \boldsymbol{\rho}_{0}^{(j)}+\Delta \boldsymbol{\rho}_{1}^{(j)}+\Delta \boldsymbol{\rho}_{2}^{(j)} \\ \frac{\boldsymbol{\rho}_{0}^{(j)}+\Delta \boldsymbol{\rho}_{1}^{(j)}-\boldsymbol{\rho}_{0}^{(j)} \Delta \boldsymbol{\rho}_{2}^{(j)} / \Delta \boldsymbol{\rho}_{1}^{(j)}}{1-\Delta \boldsymbol{\rho}_{2}^{(j)} / \Delta \boldsymbol{\rho}_{1}^{(j)}} & \end{array}\right)$,

$\left.\underset{\frac{\mathcal{I}_{0}+\Delta \mathcal{I}_{1}-\mathcal{I}_{0} \Delta \mathcal{I}_{2} / \Delta \mathcal{I}_{1}}{1-\Delta \mathcal{I}_{2} / \Delta \mathcal{I}_{1}}}{\mathcal{I}_{0}+\Delta \mathcal{I}_{1}+\Delta \mathcal{I}_{2}}\right)$

relation $^{1}$

$$
\Gamma_{L / R}=i\left[\Sigma_{L / R}^{>}-\Sigma_{L / R}^{<}\right]=i\left[\Sigma_{L / R}^{r}-\Sigma_{L / R}^{a}\right] .
$$

From the lesser/greater ballistic Green's function $g_{0}^{\lessgtr}$ one can calculate the ballistic charge densities such as

$$
\boldsymbol{\rho}_{0, e /(h)}^{(j)}=-\frac{i}{V} \int \frac{d E}{2 \pi} \operatorname{Tr}^{(j)}\left[g_{0}^{</(>)}(E)\right],
$$

where $V$ is the volume of the discretized cell and the trace operator $\operatorname{Tr}^{(j)}$ acts on the terms of the chosen local basis $(j)$. The ballistic current is given by

$$
\mathcal{I}_{0,(j)}=\frac{e}{\hbar} \int \frac{d E}{2 \pi} \operatorname{Tr}\left[\Sigma_{(j)}^{\gtrless} g_{0}^{\lessgtr}(E)-\Sigma_{(j)}^{\lessgtr} g_{0}^{\gtrless}(E)\right],
$$

with $\Sigma \underset{(j)}{\lessgtr}$ as the self-energy between slices $(j)$ and $(j+1)$ along the transport direction, $\hbar$ as the reduced Planck constant, $e$ as the electronic charge, and $\operatorname{Tr}$ as the trace operator on the whole matrix.

We now introduce the electron-phonon scattering by calculating the higher order Born series. The electron mass being much smaller compared to the ion mass ${ }^{35}$, the phonon bath is assumed to be in thermal equilibrium. Therefore, the self-energy of interaction with optical phonons can be expressed as

$$
\begin{aligned}
\Sigma_{o p}^{\lessgtr}(E) & =M_{o p}\left[N_{o p} g_{0}^{\lessgtr}(E \mp \hbar \omega)\right. \\
& \left.+\left(N_{o p}+1\right) g_{0}^{\lessgtr}(E \pm \hbar \omega)\right],
\end{aligned}
$$

where $M_{o p}$ (unit in $\mathrm{eV}^{2}$ ) describes the strength of the optical-phonon interaction and $N_{o p}=1 /\left(\mathrm{e}^{\hbar \omega / k_{\mathrm{B}} T}-1\right)$ is the phonon occupation number at the optical phonon energy $\hbar \omega$. Interactions with acoustic phonons are assumed to generate elastic processes $(E \pm \hbar w \approx E)$ and can be reduced to

$$
\Sigma_{a c}^{\lessgtr}(E)=M_{a c} g_{0}^{\lessgtr}(E),
$$


where $M_{a c}$ (unit in $e V^{2}$ ) is the intensity of the acousticphonon interaction. We then define an interaction selfenergy

$$
\Sigma_{i n t}^{\lessgtr}=i[\Sigma \underset{o p}{\lessgtr}+\Sigma \underset{a c}{\lessgtr}] .
$$

Here, the self-energies for the electron-phonon scattering are treated differently in the SCBA and LOA cases. For the SCBA, we inject these self-energies Eqs. $(23-25)$ into Eqs. $(16-17)$ and iteratively solve the following system

$$
\begin{gathered}
G_{N}^{\lessgtr}=G_{N}^{r}\left(\Sigma_{L / R}^{\lessgtr}+\Sigma_{i n t}^{\lessgtr}\left[G_{N-1}\right]\right) G_{N}^{a}, \\
G_{N}^{r}=\left[E I-H-\Sigma_{L / R}^{r}-\Sigma_{i n t}^{r}\left[G_{N-1}\right]\right]^{-1}, \\
\Sigma_{i n t}^{r}=\frac{\left[\Sigma_{i n t}^{>}-\Sigma_{i n t}^{<}\right]}{2},
\end{gathered}
$$

where $G_{0}^{r / a}=g_{0}^{r / a}$ and $G_{0}^{\lessgtr}=g_{0}^{\lessgtr}$. In the case of LOA, the non-interacting Green's function $g_{0}^{\lessgtr}$ can be used for the calculation of the self-energy in Eq. (23) as the first order LOA. However, it should be replaced by the perturbation terms $\Delta g_{N-1}$ for the higher order LOA $(N \geqslant 1)$ such that

$$
\begin{aligned}
& \Sigma_{o p, N}(E)=M_{o p}\left[N_{o p} \Delta g_{N-1}^{\lessgtr}(E \mp \hbar \omega)\right. \\
&\left.+\left(N_{o p}+1\right) \Delta g_{N-1}^{\lessgtr}(E \pm \hbar \omega)\right], \\
& \Sigma_{a c, N}^{\lessgtr}(E)=M_{a c} \Delta g_{N-1}^{\lessgtr}(E),
\end{aligned}
$$

where $\Delta g_{0}^{\lessgtr}=g_{0}^{\lessgtr}$ is the perturbation term of the firstorder LOA. This process imparts physical interpretations on the LOA meaning that first-order LOA exactly includes one electron-phonon interaction processes, secondorder LOA exactly two electron-phonon interactions, and so on.

Now that the non-interacting Green's function and the electron-phonon self-energies are defined, we return to the generalized LOA Eq. (8). This equation shows how to compute the retarded Green's function of the LOA to any order. However, to obtain the general formulation of the lesser/greater Green's function of the LOA at any order we need to apply the Langreth theorem ${ }^{7}$ to this equation. This theorem states that

$$
G^{\lessgtr}=g_{0}^{r}\left[\Sigma_{i n t}^{r} G^{\lessgtr}+\Sigma_{i n t}^{\lessgtr} G^{a}+\Sigma_{L / R}^{\lessgtr} G^{a}\right] .
$$

Noticing that $g_{0}^{r} \Sigma_{L / R}^{\lessgtr}=g_{0}^{\lessgtr}\left(g_{0}^{a}\right)^{-1}$ and that $\left(g_{0}^{a}\right)^{-1} G^{a}=$ $I+\sum_{i n t}^{a} G^{a}$, we can rewrite

$$
G^{\lessgtr}=g_{0}^{\lessgtr}+g_{0}^{r}\left(\Sigma_{i n t}^{r} G^{\lessgtr}+\Sigma_{i n t}^{\lessgtr} G^{a}\right)+g_{0}^{\lessgtr} \Sigma_{i n t}^{a} G^{a},
$$

so that the Born series is given by

$$
\begin{aligned}
\Delta g_{N}^{\lessgtr} & =\sum_{n=0}^{N-1}\left[g_{0}^{r} \Sigma_{i n t, N-n}^{r} \Delta g_{n}^{\lessgtr}\right. \\
& \left.+g_{0}^{r} \Sigma_{\text {int }, N-n}^{\lessgtr} \Delta g_{n}^{a}+g_{0}^{\lessgtr} \Sigma_{i n t, N-n}^{a} \Delta g_{n}^{a}\right],
\end{aligned}
$$

with

$$
\Delta g_{N}^{\lessgtr}=g_{N}^{\lessgtr}-g_{N-1}^{\lessgtr} .
$$

Finally, once we get the lesser/greater LOA Green's function, calculation of the current and the charge density can be performed by using Eqs. $(21-22)$

$$
\begin{gathered}
\boldsymbol{\rho}_{N, e /(h)}^{(j)}=-\frac{i}{V} \int \frac{d E}{2 \pi} \operatorname{Tr}^{(j)}\left[g_{N}^{</(>)}(E)\right], \\
\mathcal{I}_{N,(j)}=\frac{e}{\hbar} \int \frac{d E}{2 \pi} \operatorname{Tr}\left[\Sigma_{(j)}^{\gtrless} g_{N}^{\lessgtr}(E)-\Sigma_{(j)}^{\lessgtr} g_{N}^{\gtrless}(E)\right] .
\end{gathered}
$$

\section{B. Matrix Form of the Padé Approximants}

When considering higher order of currents and carrier densities, the Padé approximants in Eq. (13) can be rapidly difficult to evaluate. We therefore refer in the following to the matrix form of the Padé approximants. ${ }^{36}$

We first turn our attention to Eq. (12). By substituting the Taylor series into this expression, we obtain the following equation

$$
\begin{aligned}
\sum_{k=0}^{l+m} f_{k} z^{k} & =f_{0}+f_{1} z+f_{2} z^{2}+\cdots+f_{l+m} z^{l+m} \\
& =\frac{l_{0}+l_{1} z+l_{2} z^{2}+\cdots+l_{l} z^{l}}{1+m_{1} z+m_{2} z^{2}+\cdots+m_{m} z^{m}}
\end{aligned}
$$

We can then rearrange it and match the same order coefficients

$$
\begin{gathered}
f_{0}+\left(f_{0} m_{1}+f_{1}\right) z+\left(f_{0} m_{2}+f_{1} m_{1}+f_{2}\right) z^{2}+\cdots \\
=l_{0}+l_{1} z+l_{2} z^{2}+\cdots
\end{gathered}
$$

so that a system of $l+m+1$ linear homogeneous equations is obtained

$$
\begin{aligned}
& l_{0}=f_{0}, \\
& l_{1}-f_{0} m_{1}=f_{1}, \\
& l_{2}-f_{0} m_{2}-f_{1} m_{1}=f_{2} \text {, } \\
& l_{l}-f_{0} m_{l}-f_{1} m_{l-1}-\cdots-f_{l-1} m_{1}=f_{l}, \\
& -f_{l-m+1} m_{m}-f_{l-m+2} m_{m-1}-\cdots-f_{l} m_{1}=f_{l+1} \text {, } \\
& -f_{l-m+2} m_{m}-f_{l-m+3} m_{m-1}-\cdots-f_{l+1} m_{1}=f_{l+2} \text {, } \\
& -f_{l} m_{m}-f_{l+1} m_{m-1}-\cdots-f_{l+m-1} m_{1}=f_{l+m} .
\end{aligned}
$$

In terms of the unknown coefficients $l_{0}, \ldots, l_{l}$ and $m_{1}, \ldots, m_{m}$, these linear equations can be transformed in a matrix form as 

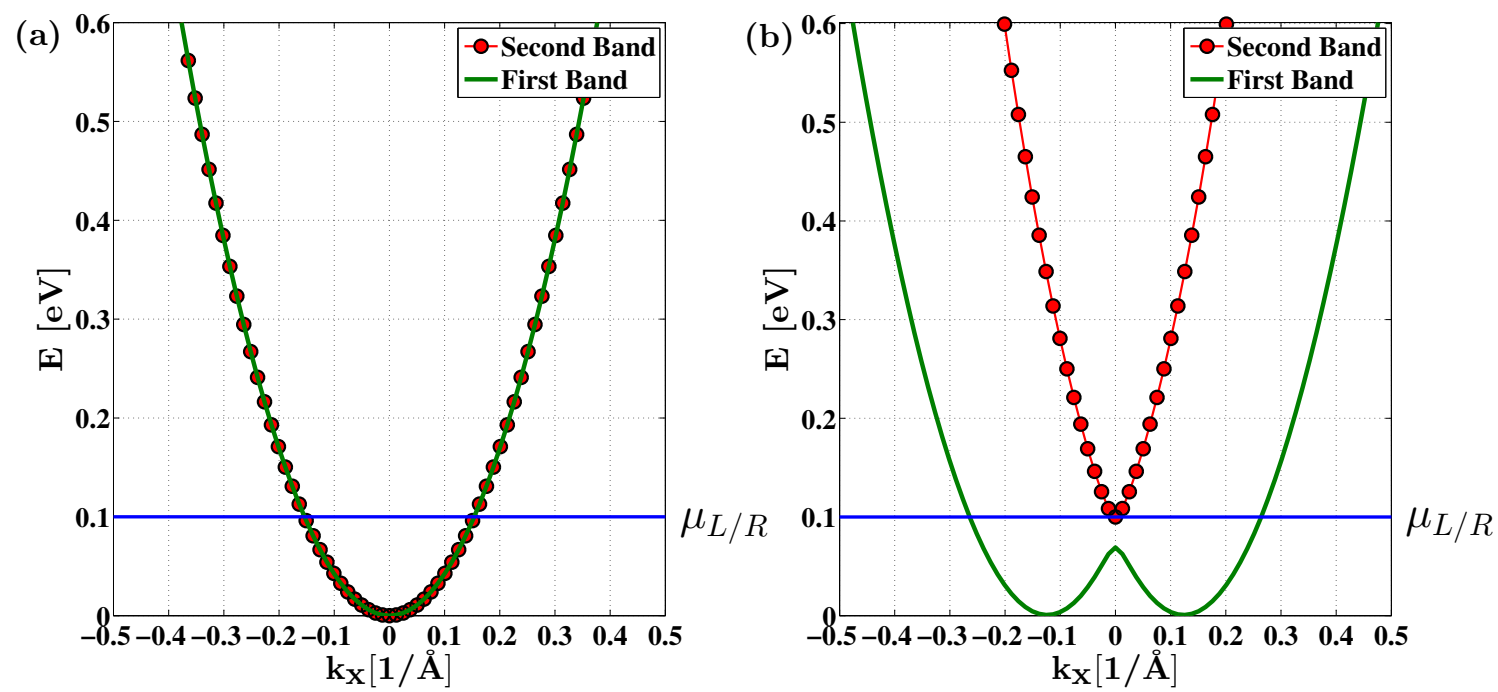

FIG. 1. (Color online) Band structures in $E-k_{x}$ diagram described by a two-band $\mathrm{k} \cdot \mathrm{p}$ Hamiltonian: (a) two-bands without coupling (TBWOC) and (b) two-bands with coupling (TBWC). The chemical potentials $\mu_{L / R}$ in the left and right reservoirs are set $0.1 \mathrm{eV}$ above the bottom of the lowest sub-band.

$$
\left[\begin{array}{cccccccc}
1 & 0 & \cdots & 0 & 0 & 0 & \cdots & 0 \\
0 & 1 & \cdots & 0 & -f_{0} & 0 & \cdots & 0 \\
\vdots & \ddots & \vdots & \vdots & & \ddots & \vdots \\
0 & 0 & \cdots & 1 & -f_{l-1} & -f_{l-2} & \cdots & 0 \\
0 & 0 & \cdots & 0 & -f_{l} & -f_{l-1} & \cdots & -f_{l-m+1} \\
0 & 0 & \cdots & 0 & -f_{l+1} & -f_{l} & \cdots & -f_{l-m+2} \\
\vdots & & \ddots & \vdots & \vdots & & \ddots & \vdots \\
0 & 0 & \cdots & 0 & -f_{l+m-1} & -f_{l+m-2} & \cdots & -f_{l}
\end{array}\right]\left[\begin{array}{c}
l_{0} \\
l_{1} \\
\vdots \\
l_{l} \\
m_{1} \\
m_{2} \\
\vdots \\
m_{m}
\end{array}\right]=\left[\begin{array}{c}
f_{0} \\
f_{1} \\
\vdots \\
f_{l} \\
f_{l+1} \\
f_{l+2} \\
\vdots \\
f_{l+m}
\end{array}\right],
$$

where we can see that it represents a $\mathbb{A} \boldsymbol{x}=\boldsymbol{b}$ linear matrix equation. By solving this equation we can easily determine the coefficients $l_{0}, \ldots, l_{l}$ and $m_{1}, \ldots, m_{m}$ for the function $F_{l / m}(z)=\frac{l_{0}+l_{1} z+l_{2} z^{2}+\cdots+l_{l} z^{l}}{1+m_{1} z+m_{2} z^{2}+\cdots+m_{m} z^{m}}$. This approach will be used in the rest of the paper.

\section{RESULTS AND DISCUSSIONS}

To validate our approach, we consider a onedimensional (1D) linear atomic chain connected to left and right reservoirs at room temperature, as widely encountered in nanoelectronic devices. We consider a twoband $\mathrm{k} \cdot \mathrm{p}$ Hamiltonian to assess the capability of such method to treat the impact of multiband transport properties, and we compute both the current and the electron density in presence of electron-phonon scattering.

The active region of the linear chain is $16 \mathrm{~nm}$ long and consists of 32 atomic sites along the $x$-direction (i.e. the lattice constant between atoms is $5 \AA$ ). In order to evaluate the impact of multi-band transport, we consider the following two-band $\mathrm{k} \cdot \mathrm{p}$ Hamiltonian $H_{k \cdot p}$ defined as

$$
H_{k \cdot p}=\left[\begin{array}{cc}
E_{2}+\frac{\hbar^{2} k_{x}^{2}}{2 m_{e 2}} & \sqrt{\frac{E_{p} \hbar^{2}}{2 m_{0}}} k_{x} \\
\sqrt{\frac{E_{p} \hbar^{2}}{2 m_{0}}} k_{x} & E_{1}+\frac{\hbar^{2} k_{x}^{2}}{2 m_{e 1}}
\end{array}\right],
$$

TABLE I. Parameters for two-band $\mathrm{k} \cdot \mathrm{p}$ Hamiltonian used in this work.

\begin{tabular}{ccc}
\hline \hline & TBWOC & TBWC \\
\hline$m_{e 1} / m_{0}, m_{e 2} / m_{0}$ & $0.9,0.9$ & $0.5,1.0$ \\
$E_{1}, E_{2}(\mathrm{eV})$ & 0,0 & $0.0691,0.1$ \\
$E_{p}$ & 0 & 0.5 \\
\hline \hline
\end{tabular}

where $E_{1}$ and $E_{2}$ are the energies of the two bands at $k_{x}=0, m_{e 1}$ and $m_{e 2}$ represent the corresponding 

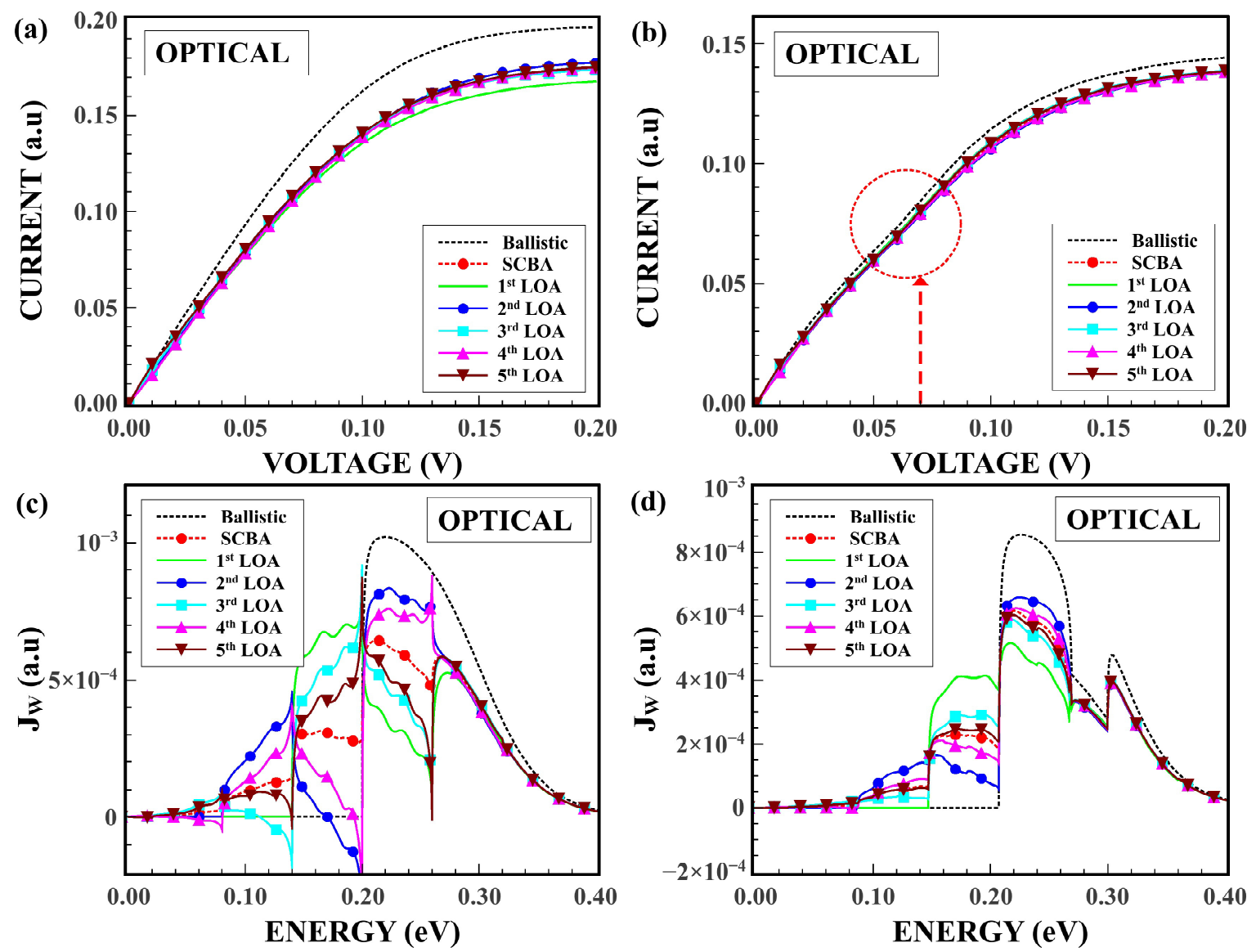

FIG. 2. (Color online) Top panel: the current-voltage characteristics in presence of optical phonon scattering $\left(M_{o p}=5 \times\right.$ $10^{-4} \mathrm{eV}^{2}$ ): (a) for TBWOC, and (b) for TBWC in the ballistic regime, the SCBA and the 5 first Lowest Order Approximations. Bottom panel: the corresponding currents spectra $\mathrm{J}_{\mathrm{w}}$ at the edge of the right active region with $V_{R L}=0.2 \mathrm{~V}$ : (c) for TBWOC, and (d) for TBWC in the ballistic regime, the SCBA and the 5 first Lowest Order Approximations. The dotted circle in Fig. 2 (b) refers to the current inflection induced by the negative curvature of the considered band structure.

effective masses and $E_{p}$ is the strength of the coupling between the two bands. By transforming $k_{x} \rightarrow-i \partial / \partial x$, we can discretize this Hamiltonian in the real space. Two different parameter sets are defined in Table I. In the first parameterization the coupling term $E_{p}$ is equal to zero and $E_{1}=E_{2}$. The two bands exactly overlap each other, and can be treated independently. Figure 1 (a) shows the resulting band structure defined as TBWOC for "TwoBand WithOut Coupling". The second parametrization, labelled TBWC for "Two-Band With Coupling", assumes a strong coupling $E_{p}$. The two bands repel each other and generate a negative curvature in the lowest one (Fig. 1(b)). It gives rise to a camel-like shape which is the characteristics, for instance, of valence band structures in Si nanowires ${ }^{37}$.

Without any applied voltages on the reservoirs $\left(\mathrm{V}_{R L}\right)$, the linear chain is in equilibrium with the left and right chemical potentials $\mu_{L / R}$ set $0.1 \mathrm{eV}$ above the bottom of the lowest sub-band (Fig. 1). A non-zero applied bias shifts the two chemical potentials by $\Delta \mu=\mu_{L}-\mu_{R}=$ $\mathrm{eV}_{R L}$ making the active region out-of-equilibrium. For the sake of simplicity a linear potential profile between the left and right reservoirs is assumed.

We first study the influence of optical phonon interactions with a frequency $\hbar w=60 \mathrm{meV}$ and consider a weak electron-phonon coupling $\left(M_{o p}=5 \times 10^{-4} \mathrm{eV}^{2}\right)$. Figures 2 (a) and 2 (b) compare the currents obtained in the ballistic regime, the SCBA and for the 5 first LOA for the two TBWOC and TBWC Hamiltonians, respectively. At $\mathrm{V}_{R L}=0.2 \mathrm{~V}$, the SCBA results show $11 \%$ current degradation for TBWOC and $4 \%$ current degradation for TBWC with respect to the ballistic currents. We clearly see that the Born series of the current rapidly converges to usual SCBA for both TBWOC and TBWC Hamiltonians. In addition, Figure 2 (b) shows that the LOA well captures the multi-band properties since the current inflection near $\mathrm{V}_{R L}=0.07 \mathrm{~V}$ induced by the negative curvature of the band structure (Fig. 1(b)) was 

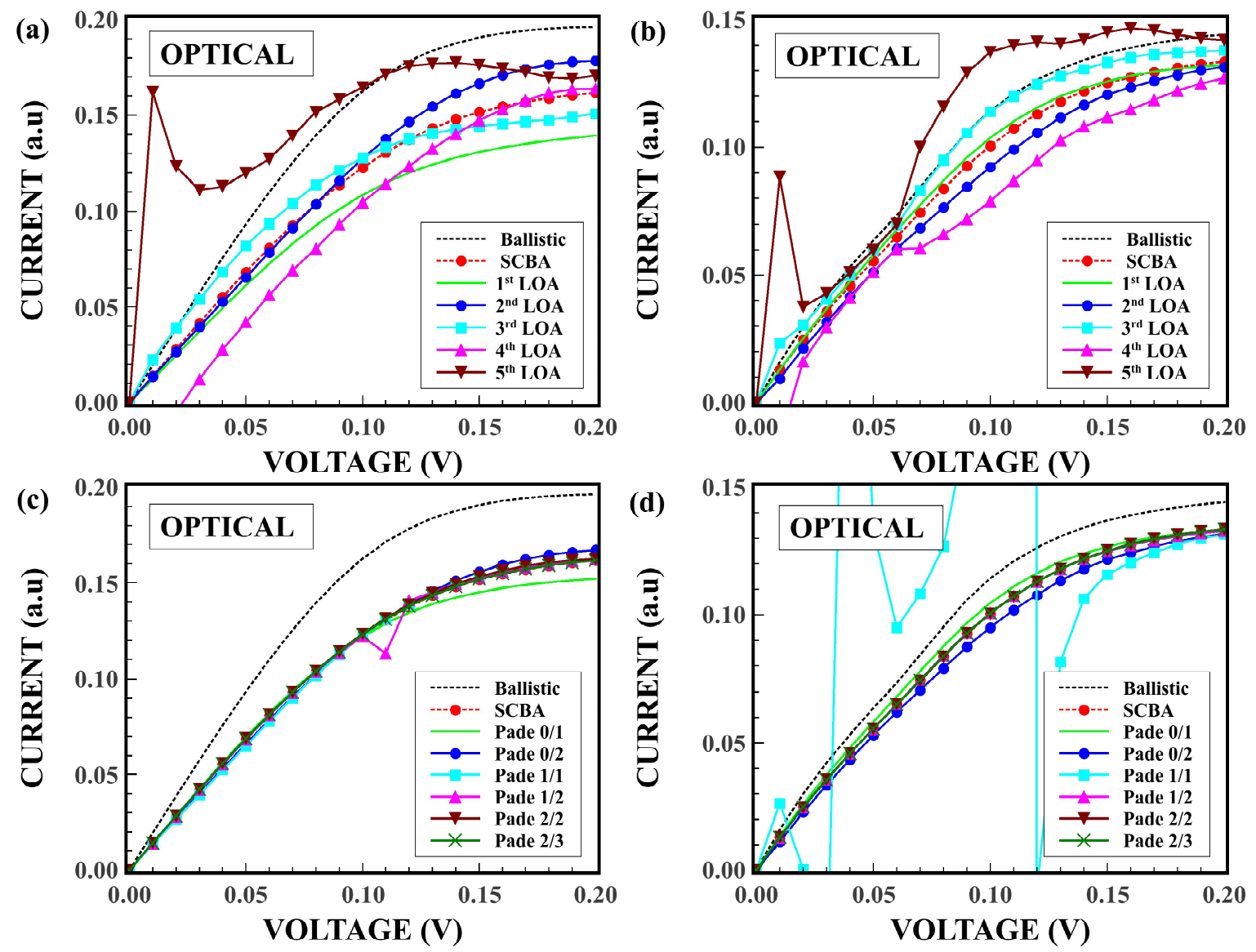

FIG. 3. (Color online) Top panel: the current-voltage characteristics in presence of optical phonon scattering $\left(M_{o p}=1 \times\right.$ $10^{-3} \mathrm{eV}^{2}$ ): (a) for TBWOC, and (b) for TBWC in the ballistic regime, the SCBA and the 5 first Lowest Order Approximations. Bottom panel: the current-voltage characteristics reconstructed based on Padé approximants: (c) for TBWOC, and (d) for TBWC. The ballistic and SCBA results are also reported.

correctly described as in the SCBA.

The corresponding spectral currents at the right-edge of the active region are plotted at $\mathrm{V}_{R L}=0.2 \mathrm{~V}$ in Figs. 2 (c) - (d) for TBWOC and TBWC, respectively. The ballistic spectrum depicts one peak for TBWOC (Fig. 2(c)) and two peaks for TBWC (Fig. 2(d)) corresponding to the band structure features shown in Fig. 1. The SCBA spectrum shows the impact of optical emission and absorption of phonons on the energy distribution of electrons for the two types of Hamiltonians. We also note that at a given order $N$ the LOA includes all the $N$ phonon scattering processes. For such a weak electronphonon coupling, the converging behavior of the series for both types of band structure confirms the relevancy of the LOA, which avoids multiple SCBA iterations without losing accuracy in the current calculation.

The situation differs when considering a larger electron-phonon coupling $\left(M_{o p}=1 \times 10^{-3} \mathrm{eV}^{2}\right)$. As shown by the current characteristics in Fig. 3, the reductions between the ballistic and the SCBA are 18
$\%$ for TBWOC and $7 \%$ for TBWC at $\mathrm{V}_{R L}=0.2 \mathrm{~V}$. Figures $3(\mathrm{a})$ and $3(\mathrm{~b})$ also show that the series of the LOA currents are now diverging with respect to SCBA values. We then apply the Padé approximants to analytically continue these divergent series. Figures 3 (c) and $3(\mathrm{~d})$ show that most of the Padé approximants successfully reproduce the results of SCBA. We also note that the Padé $1 / 1$ in Fig. $3(\mathrm{~d})$ exhibits strong singularities. These singularities result from the cancellation of the denominator of the rational expression of the Padé approximants. This behavior occurs when $\Delta \mathcal{I}_{1}$ of Eq. (15) is equal to $\Delta \mathcal{I}_{2}$. To better understand the divergence of the LOA series, Figure 4 shows the current spectra at the edge of the right active region at $\mathrm{V}_{R L}=$ $0.2 \mathrm{~V}$. We clearly see that the odd and even orders of the LOA oscillate alternatively in opposite directions, while going away from the SCBA.

We put forward our approach to check the validity for strong optical phonon scattering and then consider an electron-phonon coupling $\left(M_{o p}=3 \times 10^{-3} \mathrm{eV}^{2}\right)$. 


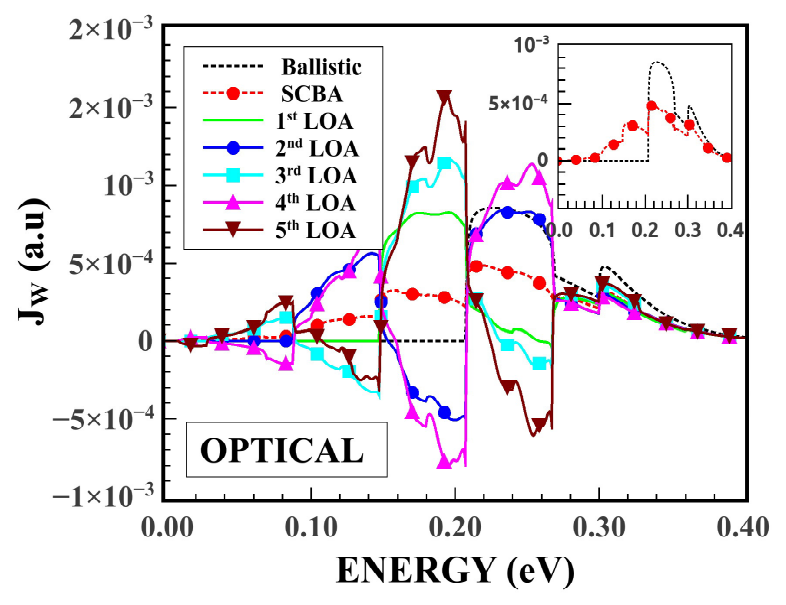

FIG. 4. (Color online) Current spectra $\mathrm{J}_{\mathrm{w}}$ of TBWC at the edge of the right active region with $\mathrm{V}_{R L}=0.2 \mathrm{~V}$ for $M_{o p}=$ $1 \times 10^{-3} \mathrm{eV}^{2}$ in the ballistic regime, the SCBA and the 5 first Lowest Order Approximations. Insets: the currents spectra for the ballistic regime and SCBA.
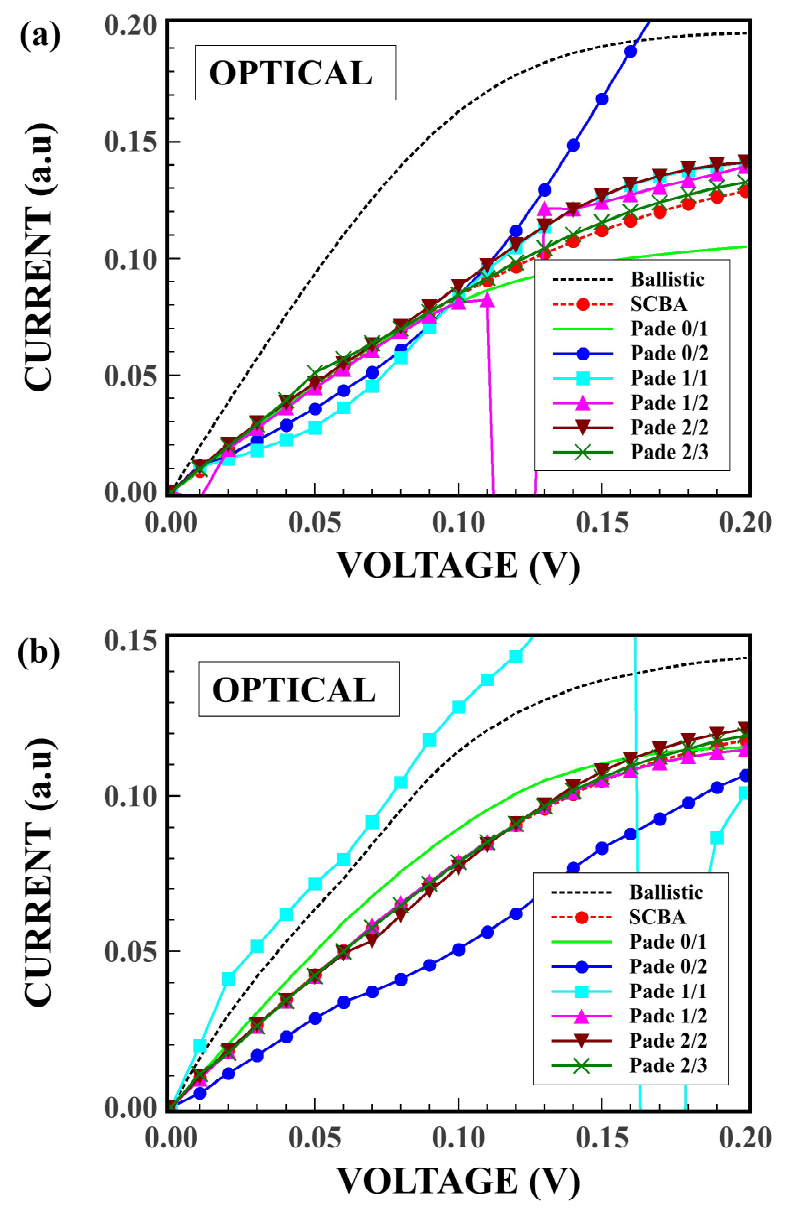

FIG. 5. (Color online) Current-voltage characteristics reconstructed based on Padé approximants in presence of optical phonon scattering $\left(M_{o p}=3 \times 10^{-3} \mathrm{eV}^{2}\right)$ : (a) for TBWOC, and (b) for TBWC with comparison to the ballistic and SCBA results.
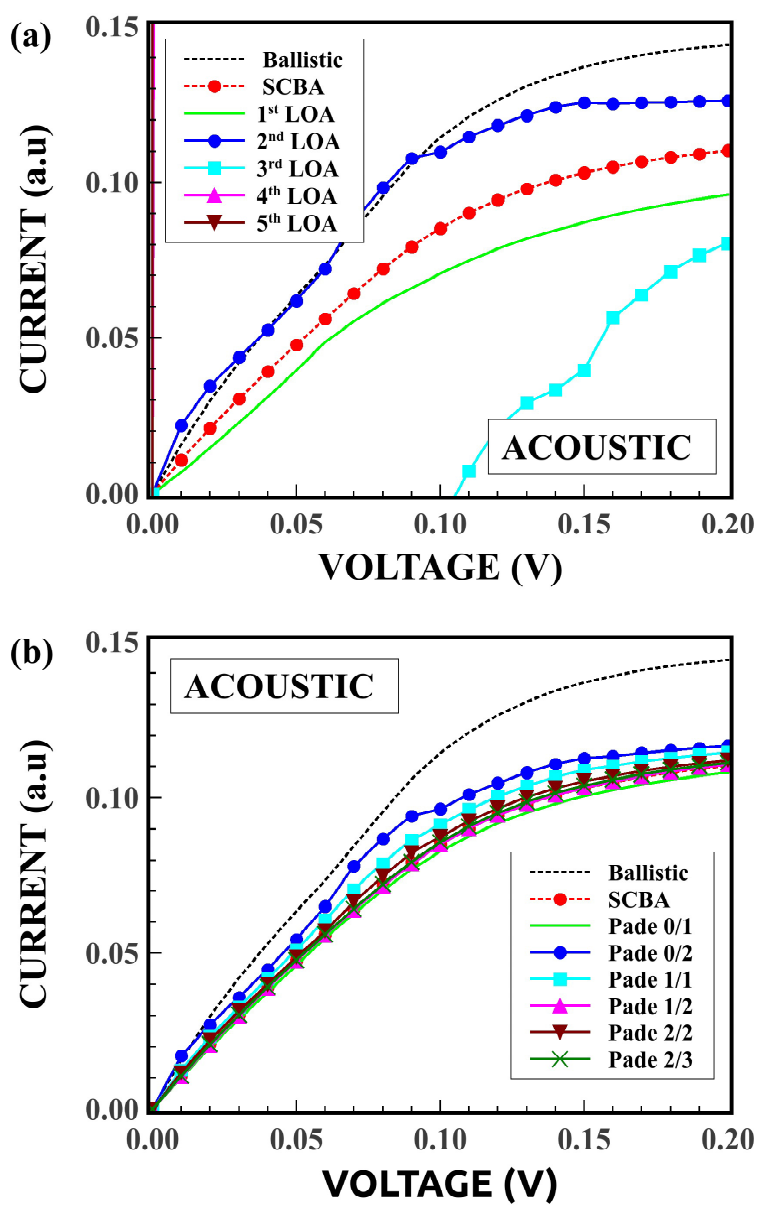

FIG. 6. (Color online) Current-voltage characteristics of TBWC in presence of acoustic phonon scattering with $M_{a c}=$ $1 \times 10^{-3} \mathrm{eV}^{2}$ : (a) in the ballistic regime, the SCBA and the 5 first Lowest Order Approximation, and (b) in the ballistic regime, the SCBA and the Padé approximants. For clear view of curves, $4^{t h}$ and $5^{t h}$ LOA are not reported in Figure 6 (a).

Figures 5 (a) and 5 (b) show that current degradations induced by the strong scattering are $34 \%$ for TBWOC and $18 \%$ for TBWC at $\mathrm{V}_{R L}=0.2 \mathrm{~V}$. All LOA series strongly diverge (not shown), and we should refer to higher order Padé approximants to successfully reconstruct these divergent series, e.g. Padé $2 / 3$ (requiring the fifth current order) for TBWOC and Padé $1 / 2$ (requiring the third current order) for TBWC.

We now focus on the impact of interactions with acoustic phonons which are defined in the present work within the elastic limit. Study of acoustic phonon scattering is an important aspect since it has been found to accelerate the divergence of the Born series in nanowire transistor ${ }^{30}$. We first consider an electron-phonon coupling $M_{a c}=1 \times 10^{-3} \mathrm{eV}^{2}$. It corresponds to a scattering which generates a $24 \%$ current degradation for TBWC at $\mathrm{V}_{R L}=0.2 \mathrm{~V}$ as shown in Fig. $6(\mathrm{a})$. Figure 6 (a) also shows that the LOA series diverges. The higher LOA currents $\left(>3^{r d} L O A\right)$ are even outside the visible range in 


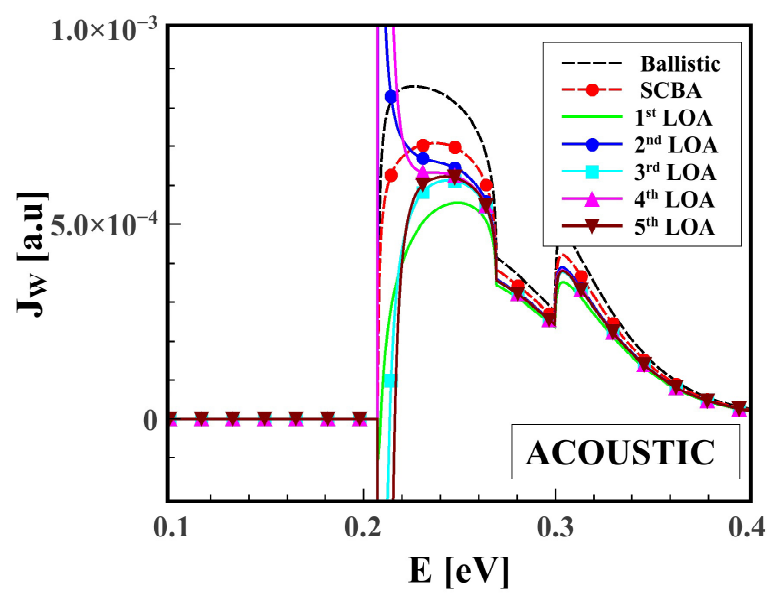

FIG. 7. (Color online) Current spectra $\mathrm{J}_{\mathrm{w}}$ of TBWC at the edge of right active region in presence of acoustic phonon scattering with $\mathrm{V}_{R L}=0.2 \mathrm{~V}$ for $M_{a c}=1 \times 10^{-3} \mathrm{eV}^{2}$ in the ballistic regime, the SCBA, and the 5 first Lowest Order Approximations.

the scale. However, application of the Padé approximants on the LOA currents is once again capable of providing SCBA results (Fig. $6(\mathrm{~b})$ ). Padé approximants are then a relevant root to reconstruct the divergent series even for a high rate of divergence. Current spectra in Fig. 7 clearly illustrate the reason of the faster divergence of the LOA with the acoustic phonon scattering than with their optical counterparts. Indeed, optical phonon scattering relaxes or excites electrons to lower or higher energies according to the number of scattering processes involved. On the other hand, acoustic phonon scattering, assumed to be elastic, does not modify the electron energy. Therefore, electrons whose energy is near the edge of the subband have a divergent density-of-states due to the Van Hove singularities (compare Fig. 4 with Fig. 7) that drastically increase the electron-phonon interactions.

When increasing the electron-phonon coupling to $M_{a c}=5 \times 10^{-3} \mathrm{eV}^{2}$, we see that acoustic phonon scattering induces a strong current degradation which is almost $60 \%$ for TBWC at $\mathrm{V}_{R L}=0.2 \mathrm{~V}$ as shown in Fig. $8(\mathrm{a})$. For such a strong scattering, all the LOA currents are highly diverging from the SCBA values. However, applying the Padé approximants to these diverging currents can still successfully reproduce SCBA results (Fig. 8(b)). In particular, we see that Padé $0 / 1,1 / 2$ and $2 / 3$ (i.e. $N / N+1$ ) which requires current calculations at the first, third, and fifth orders are more efficient to rapidly converge toward the SCBA results.

Calculation of charge density is another important aspect since it directly determines the potential profile inside the device within the Hartree approximation. We then examine the validity of the analytically continuated LOA method to calculate the charge densities in the linear chain. For the sake of clarity we do not solve the
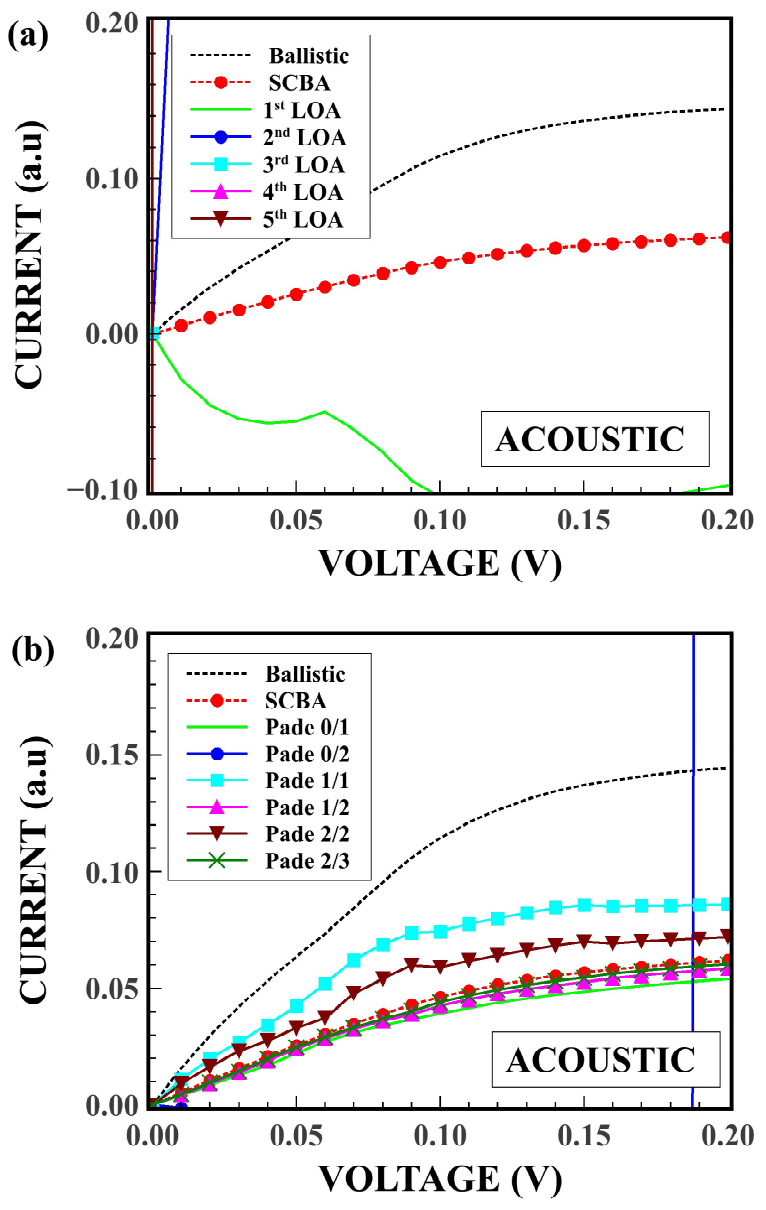

FIG. 8. (Color online) Current-voltage characteristics of TBWC in presence of acoustic phonon scattering with $M_{a c}=$ $5 \times 10^{-3} \mathrm{eV}^{2}$ : (a) in the ballistic regime, the SCBA, and the 5 first Lowest Order Approximations, and (b) in the ballistic regime, the SCBA, and the Padé approximants. For clear view of curves, $2^{\text {nd }} \sim 5^{\text {th }}$ LOA are not reported in Figure 8 (a).

Poisson equation to get a self-consistent potential. The system is first considered at equilibrium, i.e. $\mathrm{V}_{R L}=0 \mathrm{~V}$. Figure 9 shows the charge density profiles along the linear chain with the TBWOC and TBWC Hamiltonians for a weak optical phonon coupling $\left(M_{o p}=1 \times 10^{-4} \mathrm{eV}^{2}\right)$. As expected for such a bias, the charge density profile in the ballistic regime is constant. On the other hand, those obtained by the SCBA and the LOA show symmetric variations with respect to the middle of the chain. This is due to the fact that the self-energy of the electron-phonon interactions is not included in the Green's functions of the reservoirs, thus generating small reflections at the contacts that are only visible at $\mathrm{V}_{R L}=0 \mathrm{~V}$. We also observe that the charge density of the LOA series obtained with TBWC converges more slowly than the one for TBWOC due to the impact of band coupling. These results confirm that the multi-band character of the transport has an important effect on the convergence behavior of the 

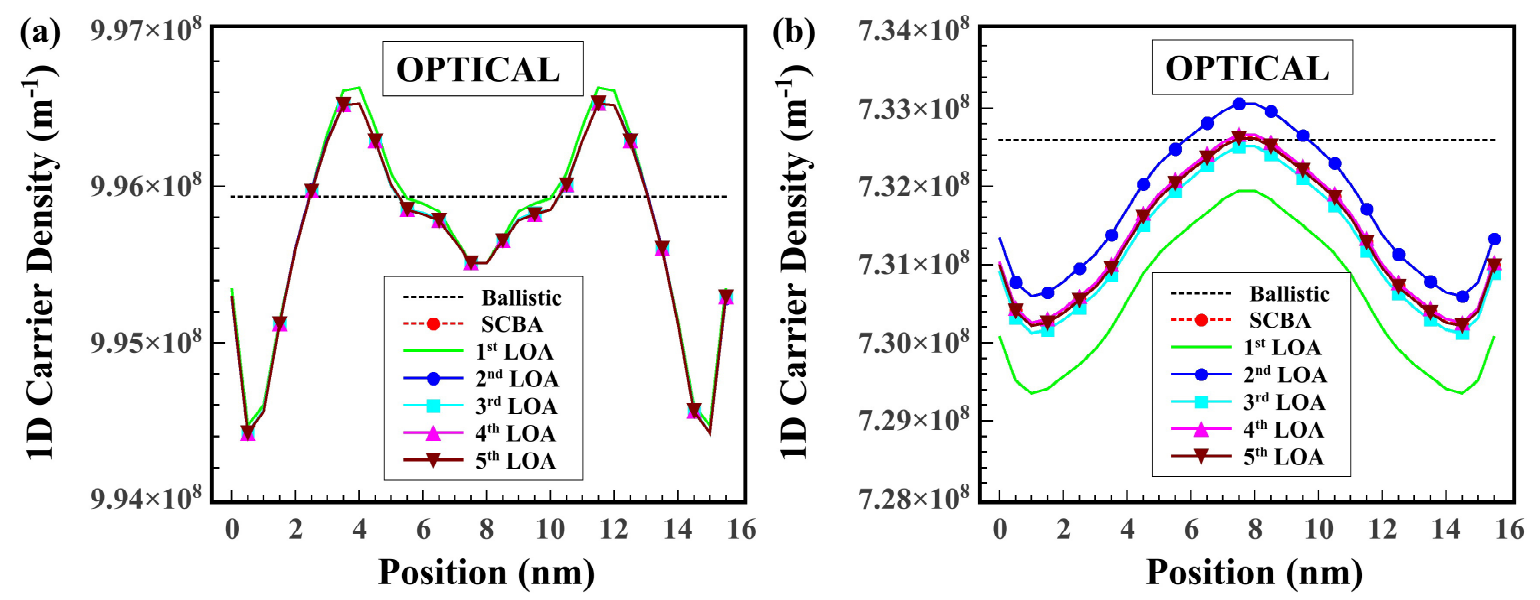

FIG. 9. (Color online) $1 \mathrm{D}$ carrier density profiles along the active region at equilibrium $\left(\mathrm{V}_{R L}=0 \mathrm{~V}\right)$ with $M_{o p}=1 \times 10^{-4} \mathrm{eV}^{2}$ : (a) for TBWOC, and (b) for TBWC in the ballistic regime, the SCBA, and the 5 first Lowest Order Approximations.
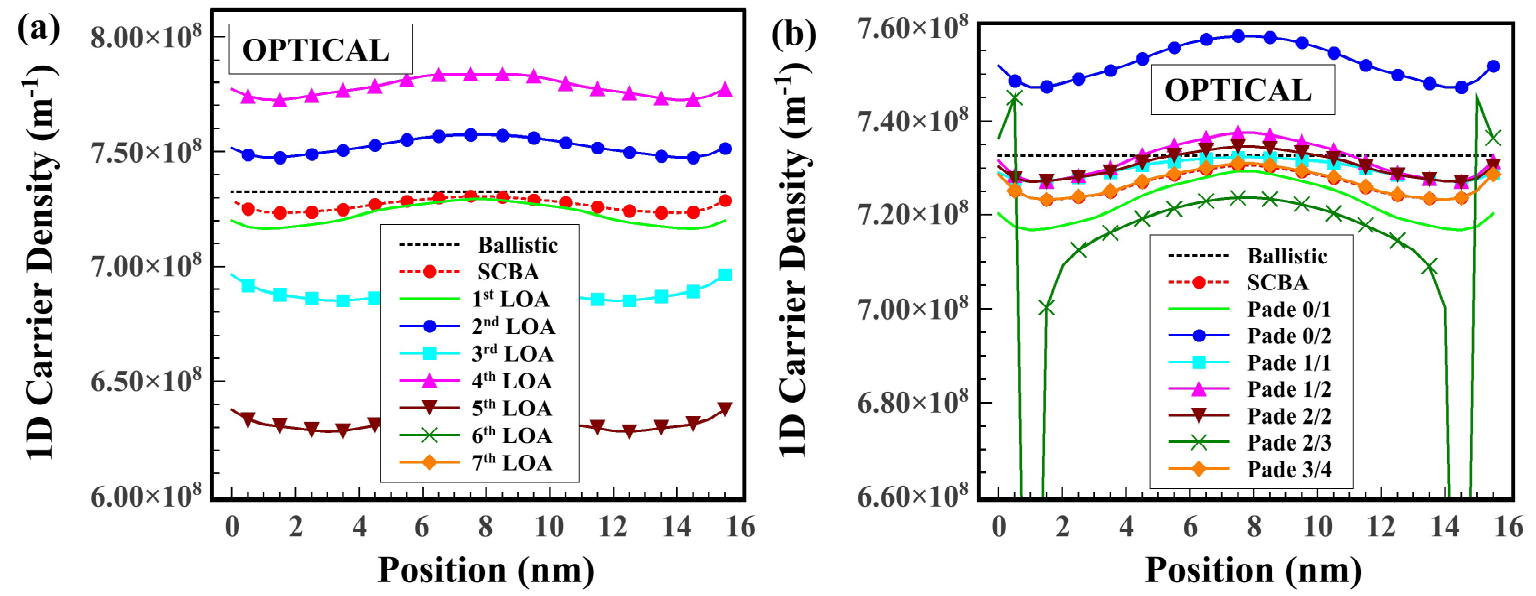

FIG. 10. (Color online) 1D carrier density profiles of TBWC along the active region at equilibrium $\left(\mathrm{V}_{R L}=0 \mathrm{~V}\right)$ with $M_{o p}=5 \times 10^{-4} \mathrm{eV}^{2}$ : (a) in the ballistic regime, the SCBA, and the 7 first Lowest Order Approximations, and (b) where LOA series are replaced by the Padé approximants. $6^{\text {th }}$ and $7^{\text {th }}$ LOA are not visible on Figure 10 (a).

series by increasing the number and the intensity of the scattering processes. However, both LOA series converge towards the SCBA result for this weak optical phonon scattering.

When considering a five times larger coupling, i.e. $M_{o p}=5 \times 10^{-4} \mathrm{eV}^{2}$, the charge density series for TBWC clearly diverges as it goes to higher LOA (Fig. 10 (a)). Here, we note that Padé approximant is again capable to analytically continue the diverging series. In that case, we need to use higher order Padé approximants $(\geqslant$ Padé $3 / 4)$ to obtain the same results as with SCBA. However, except for the Padé $0 / 2$ and $2 / 3$, the other order Padé approximants also show good results within $\sim$ $1 \%$ error to the SCBA result (Fig. $10(\mathrm{~b})$ ).

We now consider a non-zero applied bias $\mathrm{V}_{R L}=0.2$ V. Figure 11 shows the charge densities obtained for both optical and acoustic scattering with a strong cou- pling $M_{o p}=M_{a c}=1 \times 10^{-3} \mathrm{eV}^{2}$. The applied bias now generates asymmetric charge density profiles since the Poisson equation is not solved to ensure the electroneutrality of the access regions. We first note that the LOA series diverge for both types of phonons (Figs. 11 (a) and $11(\mathrm{c})$ ). However, like for the current calculations the divergence is faster for acoustic phonons than for optical ones. For the optical phonon scattering (Fig. 11 (a)), active regions near the right reservoir where the forward scattering dominates are diverging slower than the other regions. On the other hand, in the case of the acoustic phonon scattering (Fig. 11 (c)), forward scattering regions (right part) diverge much faster than back scattering regions (left part). These different behaviors of divergence are due to the different elastic and inelastic nature of the acoustic and optical phonon scattering respectively. Padé approximants ( $\geqslant$ Padé $2 / 3$ ) applied to 

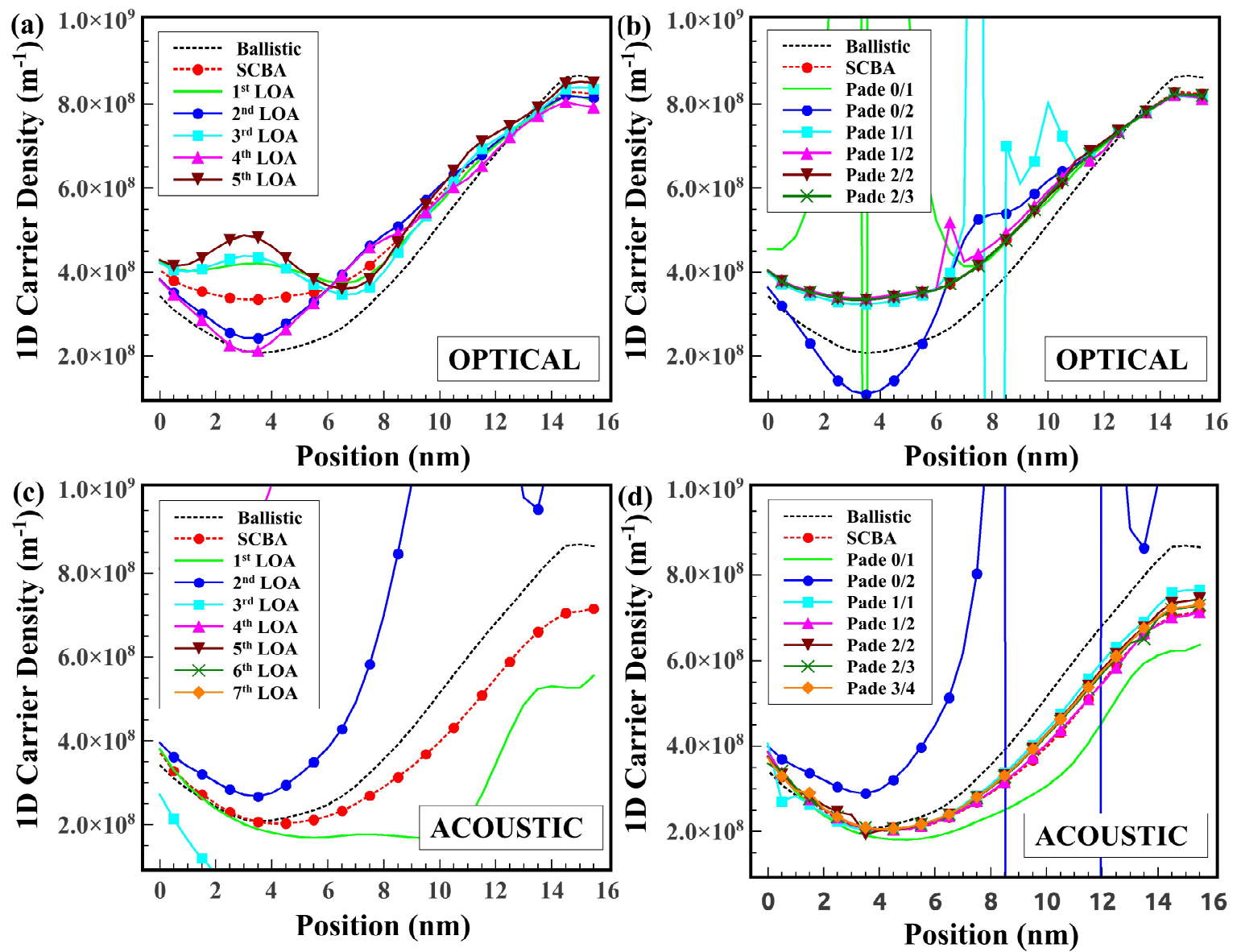

FIG. 11. (Color online) Top panel: $1 \mathrm{D}$ carrier density profiles of TBWC with $\mathrm{V}_{R L}=0.2 \mathrm{~V}$ considering optical phonon scattering with $M_{o p}=1 \times 10^{-3} \mathrm{eV}^{2}$ : (a) in the ballistic regime, the SCBA, and the 5 first Lowest Order approximations, and (b) in the ballistic regime, the SCBA, and the corresponding Padé approximants. Bottom panel: 1D carrier density profile of TBWC with $\mathrm{V}_{R L}=0.2 \mathrm{~V}$ considering acoustic phonon scattering with $M_{a c}=1 \times 10^{-3} \mathrm{eV}^{2}$ : (c) in the ballistic regime, the SCBA, and the 7 first Lowest Order approximations, and (d) in the ballistic regime, the SCBA, and the corresponding Padé approximants.

the divergent series of the charge density still reproduce the SCBA results for both types of scattering (Figs. 11 (b) and $11(\mathrm{~d}))$. Although the calculation of the charge density requires higher order Padé approximants, the analytically continuated LOA approach still shows a clear advantage to describe transport properties in quantum simulations without iterative scheme.

Finally, in order to illustrate the numerical efficiency of our approach we compare computational times at $\mathrm{V}_{R L}$ $=0.07 \mathrm{~V}$ and $0.2 \mathrm{~V}$ for the SCBA with those for the 5 first LOA (see Table II) in the case of strong optical and acoustic phonon scattering for TBWC. At $\mathrm{V}_{R L}=0.07$ $\mathrm{V}$ where the SCBA requires more than 40 iterations due to complex band structure features, all LOA calculations outperform the SCBA ones. We also see that computational times of the LOA do not significantly vary with bias, while the number of SCBA iterations depends on it. In total runtime to SCBA, it is shown that the simulation for the optical phonon scattering takes more computational times than that for the acoustic phonon one. This is due to the fact that even though the SCBA for the optical phonon scattering at high bias shows a faster convergence than for the acoustic one, at low bias $\left(\mathrm{V}_{R L} \leqslant 0.05\right.$ $\mathrm{V})$ it does not converge after 200 iterations with $<2 \%$ error. In addition, we see that calculating $1^{\text {st }} L O A$ coupled with the Padé $0 / 1$ is 40 times faster than the SCBA. Calculation of $5^{\text {th }} L O A$ with Padé $2 / 3$ is still efficient with respect to the SCBA. We then expect that for devices where strong scattering processes are dominant, our approach is much more powerful. Very recently Mera et al. have reported in Ref. 38 an approach based on Gauss hypergeometric resummation to model the Stark effect in the hydrogen atom. Even more recently ${ }^{39}$, they applied this technique to the current calculation in presence of optical phonon scattering in nanostructures. They consider an opposite transport regime as in the present 
TABLE II. Comparison of computational times for TBWC at $\mathrm{V}_{R L}=0.07 \mathrm{~V}$ and $0.2 \mathrm{~V}$ for the SCBA with those for 5 first Lowest Order Approximations in presence of strong optical and acoustic phonon scattering with $M_{o p}=M_{a c}=5 \times 10^{-3} \mathrm{eV}^{2}$. Total computational times for all bias points are also compared.

\begin{tabular}{cccccccc}
\hline \hline & & SCBA & $1^{\text {st }}$ LOA & $2^{\text {nd }}$ LOA & $3^{\text {rd }}$ LOA & $4^{\text {th }} \mathrm{LOA}^{\text {th }} \mathrm{LOA}$ \\
\hline Runtime (s) & Optical & 744 & 17 & 39 & 70 & 111 & 162 \\
at $\mathrm{V}_{R L}=0.07 \mathrm{~V}$ & Acoustic & 738 & 16 & 33 & 59 & 91 & 132 \\
\hline Runtime (s) & Optical & 191 & 17 & 39 & 69 & 109 & 158 \\
at $\mathrm{V}_{R L}=0.2 \mathrm{~V}$ & Acoustic & 535 & 15 & 33 & 59 & 131 & 132 \\
\hline Total runtime (s) & Optical & 13934 & 357 & 819 & 1470 & 2331 & 3402 \\
& Acoustic & 12166 & 336 & 693 & 1240 & 1911 \\
\hline \hline
\end{tabular}

work, i.e. quasi-localized states weakly coupled to electron reservoirs. In this particular regime, the authors have shown that a fourth order hypergeometric approach was able to outperform Padé approximants $1 / 1$ and $2 / 2$. It then represents a relevant technique to treat inelastic scattering in nanodevice quantum simulations. Further investigations should be done along that direction for other general transport regimes.

\section{CONCLUSIONS}

In this work we presented a numerically efficient approach, coupling the Born series of a given observable with the Padé approximant technique, to treat inelastic scattering in nanostructures. The approach has been applied to the treatment of electron-phonon interactions and has been systematically compared to the usual, time consuming, self-consistent Born approximation. As a fair illustration of systems encountered in nanoelectronics, we considered a linear chain in presence of both acoustic and optical phonons. Two-band k.p Hamiltonians have been implemented to analyze the capability of the approach to capture multi-band scattering. Our results first show that the method is efficient to calculate the current values. Indeed, the Padé approximants constructed with the fifth order terms in interactions are capable to provide the SCBA values for a large variety of coupling intensities and biases. The approach has been also successfully applied to the calculation of the charge density, for which it has been found that the convergence is more difficult to achieve, requiring calculations of higher orders of the electron density Born series. The presented technique remains a relevant alternative to avoid the iterative scheme of the SCBA. Its main advantage is that it can be easily generalized systematically to any order of the interaction and any Padé table. It can then be rapidly implemented in any SCBA quantum transport code. Its main drawback is that second, third, and higher order perturbation terms require important number of matrix operations and a large amount of memory storage. Their implementation in three-dimensional atomistic quantum transport codes to model realistic nano-structures is still numerically challenging and difficult to overcome. However, our results showed that $N / N+1$ Padé approximants are more reliable in reconstructing the divergent current and density series in the considered system and the simple 0/1 Padé approximant should still be of relative interest in this context.

\section{ACKNOWLEDGEMENT}

This work was supported by the NOODLES contract (ANR-13-NANO-0009) funded by the ANR-French National Research Agency. The author Y. Lee would like to thank the $A^{*}$ MIDEX for financial support. The authors would like to thank Lidiya Yushchenko from ISENToulon for fruitful discussion on Padé approximants.
* youseung.lee@im2np.fr

† marc.bescond@im2np.fr

1 S. Datta, Electronic Transport in Mesoscopic Systems (Cambridge University Press, Cambridge, U.K., 1995).

2 M. V. Fischetti, Phys. Rev. B 59, 4901 (1999).

3 S. Datta, Superlattices Microstruct. 28, 253 (2000).

4 D. Querlioz, J. Saint-Martin, V.-N. Do, A. Bournel, and P. Dollfus, IEEE Trans. Nanotechnol. 5, 737 (2006).

5 J. Sellier and I. Dimov, J. Comp. Phys. 280, 287 (2015).

6 J. Sellier and I. Dimov, J. Comp. Electron. 14, 907 (2015).

7 H. Haug and A.-P. Jauho, Quantum Kinetics in Transport and Optics of Semiconductors, vol. 123 of Springer Series in Solid-State Sciences (Springer, Berlin, New York, 1996).

${ }^{8}$ Y. Yu, H. Zhan, Y. Wei, and J. Wang, Phys. Rev. B 90, 075407 (2014).

9 Y.-S. Liu, H. Chen, X.-H. Fan, and X.-F. Yang, Phys. Rev. B 73, 115310 (2006).

10 A. Ghosh, P. Damle, S. Datta, and A. Nitzan, MRS Bulletin 29, 391 (2004).

11 A. Szabó, R. Rhyner, and M. Luisier, Phys. Rev. B 92, 035435 (2015).

12 R. Lake, R. C. B. G. Klimeck, and D. Jovanovic, J. Appl. Phys. 81, 7845 (1997). 
13 A. Svizhenko and M. P. Anantram, IEEE Trans. Electron Devices 50, 1459 (2003).

14 S. Jin, Y. J. Park, and H. S. Min, J. Appl. phys. 99, 123719 (2006).

15 M. Luisier and G. Klimeck, Phys. Rev. B 80, 155430 (2009).

16 M. Luisier, Appl. Phys. Lett. 98, 032111 (2011).

17 M. Luisier, Electron Dev. Lett. 32, 1686 (2011).

18 N. Cavassilas, F. Michelin, and M. Bescond, J. Appl. Phys. 109, 073706 (2011).

19 H. Carrillo-Nuñez, M. Bescond, N. Cavassilas, E. Dib, and M. Lannoo, J. Appl. Phys. 116, 164505 (2014).

${ }^{20}$ U. Aeberhard and R. Morf, Phys. Rev. B 77, 125343 (2008).

${ }^{21}$ U. Aeberhard, J. Comp. Electron. 10, 394 (2011).

22 N. Cavassilas, F. Michelini, and M. Bescond, Appl. Phys. Lett. 105, 063903 (2014).

23 J. M. Luttinger and J. C. Ward, Phys. Rev. 118, 1417 (1960).

24 J. M. Luttinger, Phys. Rev. 119, 1153 (1960).

${ }^{25}$ H. Mera, M. Lannoo, C. Li, N. Cavassilas, and M. Bescond, Phys. Rev. B 86, 161404 (2012).

26 H. Mera, M. Lannoo, N. Cavassilas, and M. Bescond, Phys. Rev. B 88, 075147 (2013).

27 G. H. Hardy, Divergent Series (Chelsea, New York, 1991).
28 J. G. A. Baker and P. Graves-Morris, Padé Approximants (Cambridge University Press, Cambridge, 1996).

29 N. Cavassilas, M. Bescond, H. Mera, and M. Lannoo, Appl. Phys. Lett. 102, 013508 (2013).

30 M. Bescond, C. Li, H. Mera, N. Cavassilas, and M. Lannoo, J. Appl. Phys. 114, 153712 (2013).

31 G. Baym and L. P. Kadanoff, Phys. Rev. 124, 287 (1961).

32 D. Shanks, J. Math. Phys. 34, 1 (1955).

33 C. T. Fike, Computer Evaluation of Mathematical Functions (Prentice-Hall, Inc., Englewood Cliffs, New Jersey, 1968).

${ }^{34}$ D. K. Ferry and S. M. Goodnick, Transport in Nanostructures (Cambridge University Press, Cambridge, U.K., 1997).

35 A. L. Fetter and J. D. Walecka, Quantum Theory of ManyParticle Systems (McGraw-Hill, San Francisco, 1971).

36 M.Vajta, in Proceedings of the 3rd TEMPUS INTCOM Symposium on Intelligent Systems in Control and Measurement (2000) p. 53.

37 Y. M. Niquet, A. Lherbier, N. H. Quang, M. V. FernandezSerra, X. Blase, and C. Delerue, Phys. Rev. B 73, 165319 (2006).

38 H. Mera, T. G. Pedersen, and B. K. Nikolic, Phys. Rev. Lett. 115, 143001 (2015).

39 H. Mera, T. G. Pedersen, and B. K. Nikolic, http://arxiv.org/pdf/1512.06846.pdf (2016). 